\title{
Changing climate and the COVID-19 pandemic: more than just heads or tails
}

\author{
Climate change can both facilitate zoonotic spillovers and have an effect on transmission chains. These effects, \\ alongside human behavior and awareness, need to be integrated in pandemic forecasting models.
}

\author{
Xavier Rodó, Adrià San-José, Karin Kirchgatter and Leonardo López
}

$\Lambda$ fter it emerged in China and rapidly ravaged Europe and the USA in a catastrophic first wave, it seemed a matter of time before the COVID-19 pandemic would harmfully strike low- and middle-income countries with limited resources. Despite its deadly toll in places such as India or Brazil, where nonetheless the disease did not spark as rapidly as it did in Europe-i.e., the effective reproduction number remained low-tropical countries remained relatively untouched compared with those in temperate regions ${ }^{1}$.

This latitudinal heterogeneous spread was initially attributed to the fact that tropical countries have in general younger populations, lower densities, acquired immunity and prior preparedness after previous pandemics ${ }^{2}$. At this stage of the pandemic, additionally, climate has not yet appeared as a recognized driver of the coronavirus SARS-CoV-2, and mechanistic understanding of the virus' climatic sensitivities remains limited ${ }^{3}$. But what are the stages in the zoonotic spillover process at which climate forcing is to be expected? And, more generally, what is the effect of climate on disease emergence and spread?

\section{Climate change and the emergence of new pathogens}

Zoonotic spillover is the multilevel process by which pathogens (e.g., SARS-CoV-2, Ebola virus, human immunodeficiency virus and avian influenza viruses) manage to overcome a series of natural barriers ${ }^{4}$ and infect other animal species ${ }^{5}$. The zoonotic spillover theory, taking into account the complexities of eco-epidemiological processes, specifies the types of barriers that have to be overcome in each situation ${ }^{4}$. Understanding which of these barriers can be affected by climate-either suppressed or enhanced, thereby facilitating spillover or buffering spillover, respectively-will be crucial for anticipating how climate change will affect these cross-species pathogen transmissions.

On the direct side, climate can facilitate a pathogen's survival, development and dissemination and thus ease spillover. However, it is unlikely that in this context climate will have a critical role in massively promoting the appearance of new spillover events. On the indirect side, instead, the effects of climate are much wider and far more complex. Climate change, superimposed onto a dramatic anthropogenic alteration of ecosystems, is leading to a gradual substitution of species, shrinking of ecosystems and decrease in species diversity ${ }^{6}$. These trend-like changes can clearly lead to spillovers in different ways and to closer and more-general encounters between wildlife and humans (Fig. 1a,b). However, climate extremes, acting on a much shorter timescale, can also directly or indirectly affect the frequency and intensity of forest fires, droughts, floods, famines and migrations, equally acting as point stressors and exerting intense zoonotic pressure ${ }^{7}$. An example might be epidemics of yellow fever virus in non-human primates in the Brazilian Atlantic Forest that began in 2016. There, the howler monkeys (Alouatta species) are highly susceptible to infection with yellow fever virus, and epizootic diseases driven by climatic anomalies often precede human cases, in epidemics that mirror zoonotic waves ${ }^{8,9}$ (Fig. 1c). Despite this facilitating role, climate should be seen mainly as a necessary but not sufficient factor that contributes to disease emergence.

\section{Impact of climate in transmission chains}

The mechanistic pathway by which climate can directly affect spillover can dramatically contribute to inter-human transmission. For example, it seems well established that in the emergence of the sixth cholera pandemic at the turn of the 20th century, the takeover by the classical strain was facilitated by a concurrent large climate anomaly in 1905 (e.g., extreme flooding followed by very cold months $)^{10}$. Subsequent strain replacements during the 20th century happened simultaneously with other large climate anomalies. However, whereas the former is true, the converse did not always apply.
In fact, in many years, Bangladesh experienced more-intense climatic anomalies that were not accompanied by replacements of cholera strains. Therefore, whereas climate stability seems to be appropriate for the enhancement of continuous transmission by the evolutionary differentiation of pathogens ${ }^{11}$, climate extremes may also promote strain selection by exposing new niches for colonization, thus favoring takeover or spillover if outperforming strains are present in their environmental (or reservoir) hosts ${ }^{12}$.

However, after inspection of climate change globally, an interesting and heterogeneous picture emerges in which the tropics showcase the intensification of the hydrological cycle associated with the rise in global temperature (Fig. 1a, trends in absolute humidity (AH)). As the atmosphere absorbs more heat due to the increase in greenhouse-gas concentrations, its precipitable water content similarly increases (by approximately $7 \%$ per additional degree of warming). Interestingly, Central Africa and Southeast Asia concentrate the largest positive trends in $\mathrm{AH}$, of $3 \mathrm{~g} / \mathrm{m}^{3}$ and above, while no major change has occurred as a whole in the Amazon basin, Central Asia or China. The 30-year climatological differences in both rainfall (Fig. 1b, left) and mean temperature (Fig. 1b, right) in 1960-1989 versus 1990-2019 are presented here for Wuhan (Fig. 1b, top), the Congo (Fig. 1b, middle) and the Amazon basin (Fig. 1b, bottom). Clear rises in mean temperature dominate in both the Amazon and the Congo areas, with substantial changes in precipitationalbeit smaller-also occurring in these same regions. Those larger differences illustrate the varying impacts of global warming worldwide during assessment of the relationship between climate change and disease emergence.

The tropical areas contain the largest diversity in mammal species (Fig. 1d, color scale), also regarding the main taxonomic groups that bear zoonosis (e.g., a maximum in the diversity of chiropteran species in 

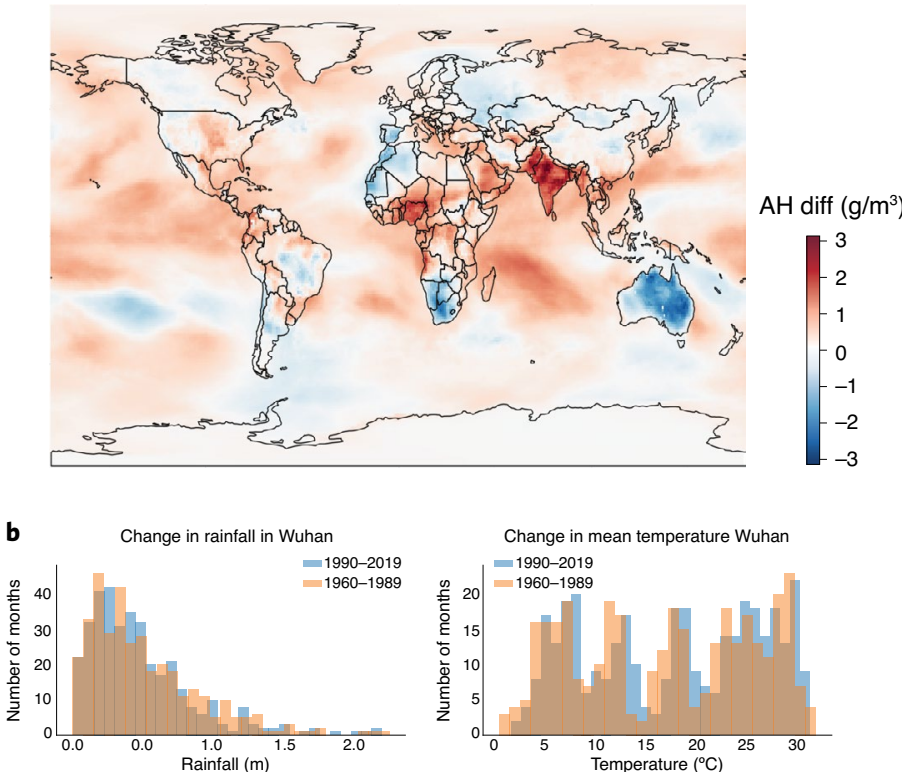

Change in rainfall in North DRC

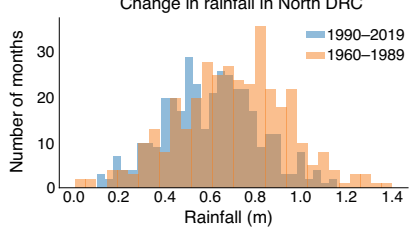

Change in rainfall in Amazonia

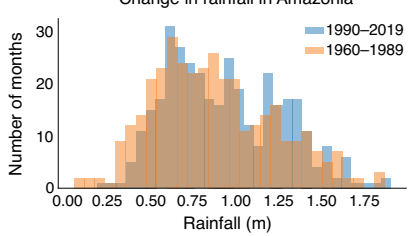

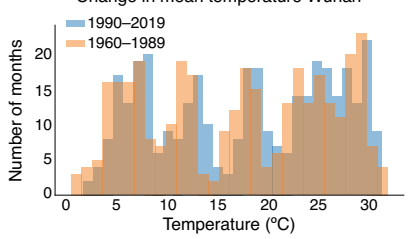

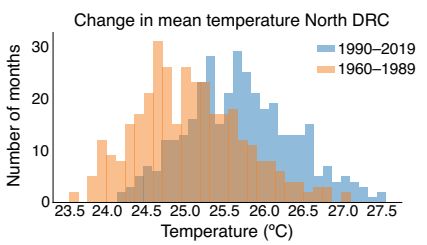

Change in mean temperature Amazonia

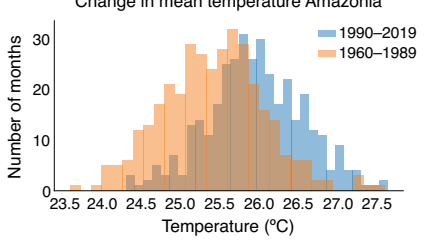

Change in mean temperature Wuhan

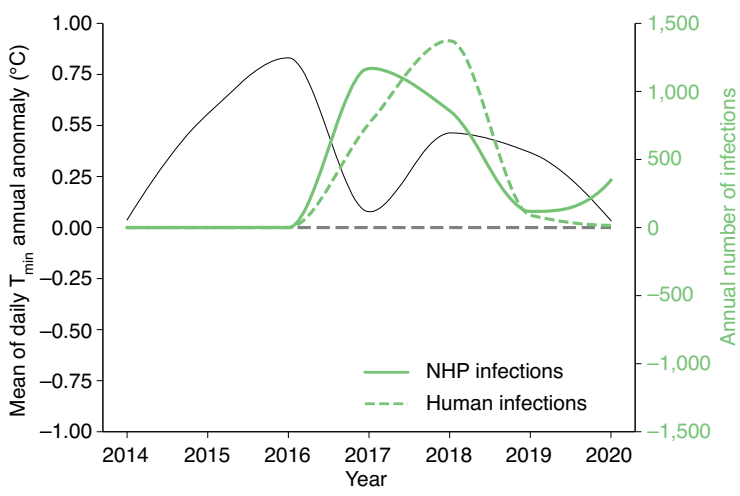

d

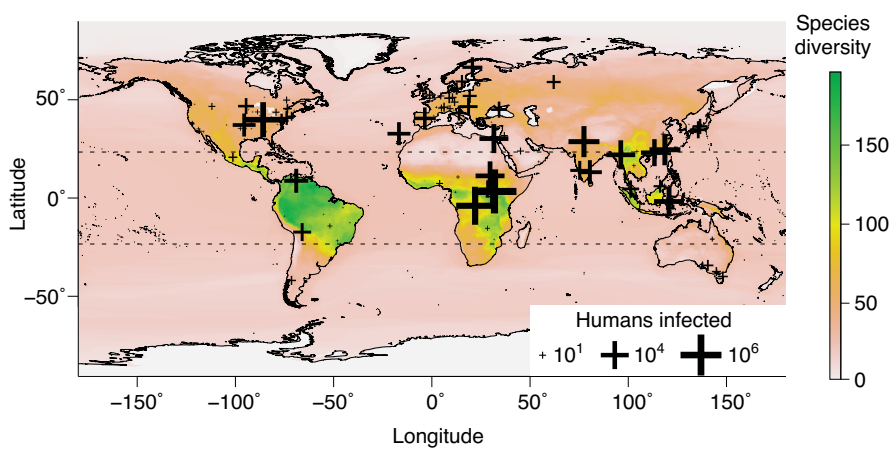

Fig. 1 | Climate change and regional zoonotic spillovers. a, Average change in AH ('AH diff'; in $\mathrm{g} / \mathrm{m}^{3}$; key) worldwide in the period 1989-2019, retrieved from the Copernicus climate data store. b, Change in mean monthly temperature (right column) and precipitation (left column) during the periods 1960-1989 and 1990-2019 (key), in a $3 \times 3$ grid centered on Wuhan, China (top: latitude, 30; longitude, 113), Sub-Saharan Africa (middle (DRC, Democratic Republic of the Congo): latitude, $1^{\circ}$; longitude, $1^{\circ}$ ), and Amazonia (bottom: latitude, $-4^{\circ}$; longitude, $-67^{\circ}$ ). Data retrieved from ERA5 (fifth-generation European Centre for Medium-Range Weather Forecasts reanalysis) through the Copernicus climate data store. c, Mean of annual anomalies in the daily minimum temperature $\left(T_{\min }\right)$ in the period 1990-2019 (gray solid line), at the Atlantic Forest (as a $3 \times 3$ grid centered at latitude $-46^{\circ}=$ and longitude $-22^{\circ}$ ), retrieved from the ERA5 reanalysis and obtained through the Copernicus climate data store, plotted with the smoothed annual number of infections in humans and non-human primates (NHP) (green lines; inset key) in the states of Espírito Santo, Rio de Janeiro, Minas Gerais, São Paulo, Paraná and Santa Catarina, retrieved from the Brazilian National Ministry of Health (https://www.gov.br/saude/pt-br/assuntos/saude-de-a-a-z-1/f/febre-amarela). d, Zoonotic emergencies in the period 1940-2013, provided by the Emerging Infectious Diseases Repository (https://eidr.ecohealthalliance.org), size-adjusted by the total number human infections that resulted from them (inset key), superimposed onto a map of mammal species richness (key, right margin) obtained from SEDAC (https://sedac.ciesin. columbia.edu/data/set/species-global-mammal-richness-2015). Publ. note: Springer Nature is neutral about jurisdictional claims in maps.

South America $\left.{ }^{13}\right)$. A linear relation between the number of zoonotic spillovers and the diversity and abundance of reservoirs has been described (e.g., in tropical regions ${ }^{14}$ ). An enhanced number of spillover events should therefore be expected to be randomly distributed year-round in the tropics as well, as food markets similarly exist in all these regions. The latitudinal distribution of spillovers, however, instead shows a clear maximum between $30^{\circ}$ north and $50^{\circ}$ north, with both direct transmission and airborne transmission dominating, in terms of disease burden, in those same latitudes but not in the tropics (Fig. 2a). Despite the considerable uncertainties and limitations that exist in zoonosis databases, these results seem stable when weighted against population densities as well.

The distribution of zoonotic outbreaks that have occurred since 1940 indicates that larger outbreaks in terms of disease incidence (for major vector-borne diseases) took place in both tropical Africa and Southeast Asia (Fig. 1d, crosses). In contrast, differences are evident for South America, which seems to be largely under-represented. As for disease emergence, data on zoonotic spillover events spanning the period 1940-2013 again show a maximum in the $1980 \mathrm{~s}$ and 1990s, centered in the temperate latitudes of the northern hemisphere, followed by a slow but persistent decrease afterward that is not mimicked by global warming ${ }^{15}$. 


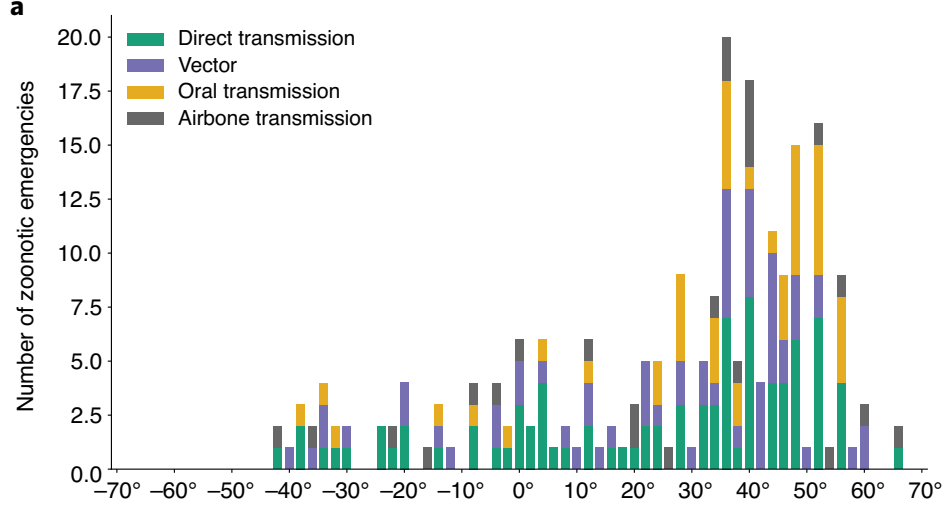

Latitude
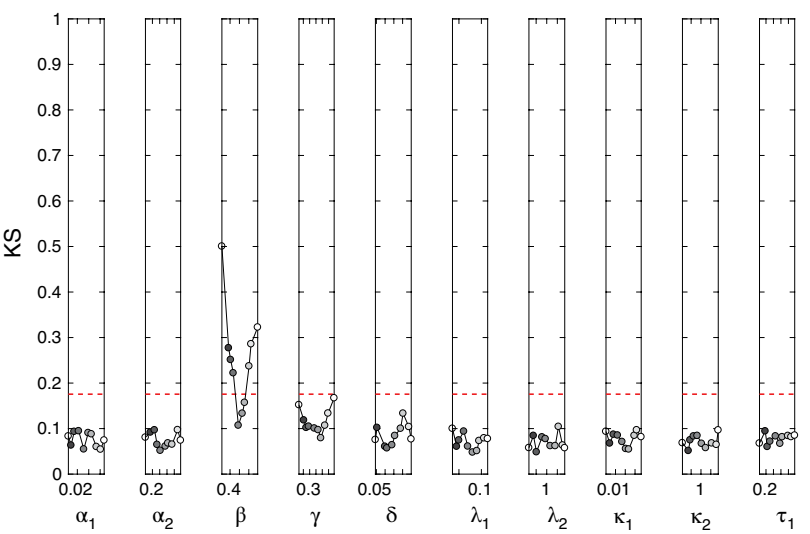

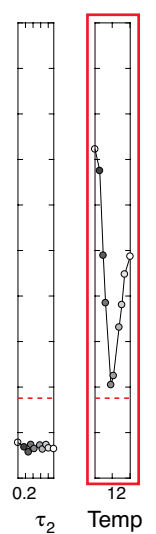

C

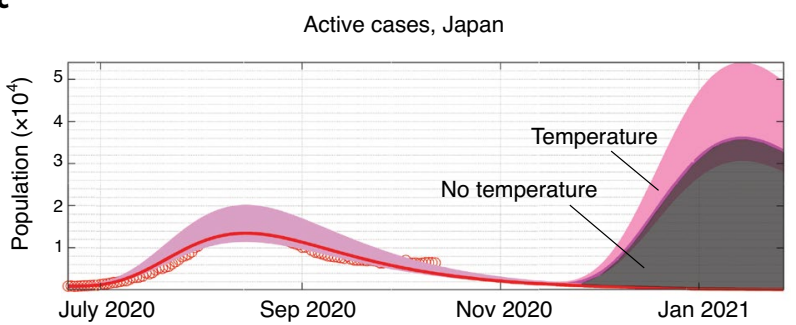

Recovered, Japan

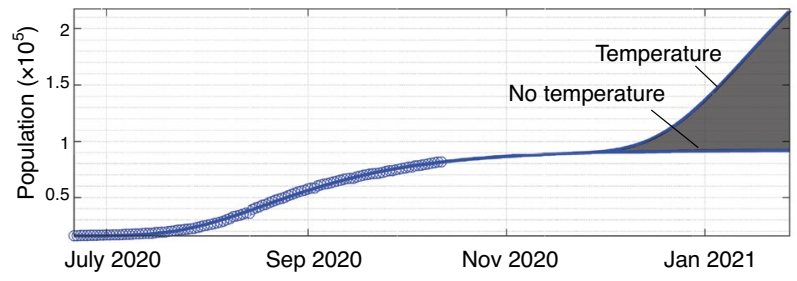

Deaths, Japan

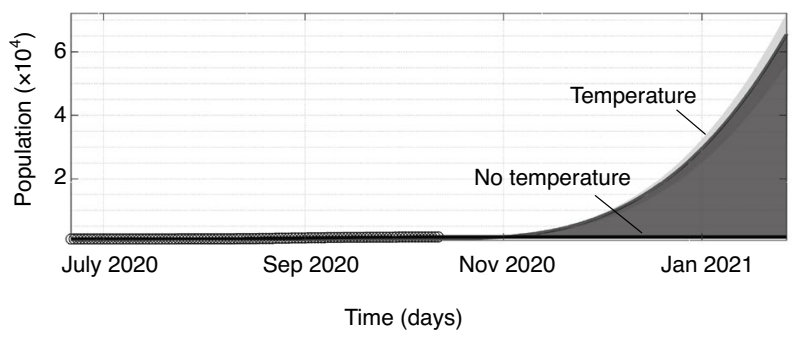

Fig. 2 | Effects of climate on respiratory zoonosis. a, Zoonotic emergencies (vertical axis) by latitude (horizontal axis) and transmission pathway (key).

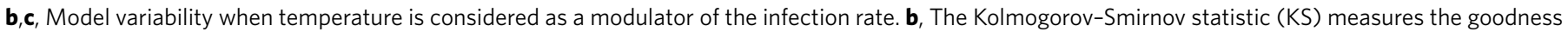
of the model in terms of how close to a normal distribution the variability indices (horizontal axis) are. The dashed red horizontal line is the critical value of the KS statistic at a confidence level of 0.05 . Infection rate is seasonal, and parameters above the confidence level are considered sensitive to the model's

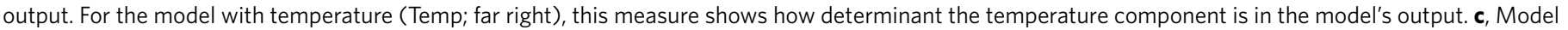
dynamics for constant and seasonal infection rates in Japan. The shaded area between the curves shows, for the number of active cases (top), recovered

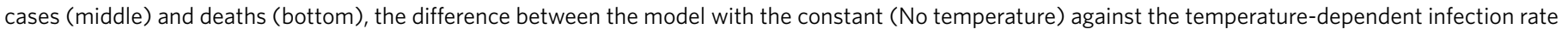

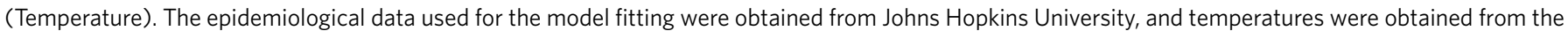

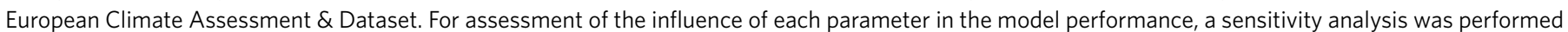
by a moment-independent global-sensitivity-analysis method (the 'PAWN' method).

\section{Respiratory zoonoses}

Special attention should be paid to the region around South China, where seasonal influenza epidemics have resulted in a surge in respiratory infections every year (e.g., $87 \%$ of the most successful, globally spreading strains of the influenza virus subtype H3N2 from 2000 to 2010 originated there $^{16}$ ) and where major outbreaks of severe acute respiratory syndrome (SARS) and COVID-19 also initially emerged. It might therefore seem counterintuitive that successful zoonotic spillovers characterized by respiratory routes of transmission are concentrated mostly during winter in the northern hemisphere, and in particular in and around China and nearby countries. However, we also note that among all the regional biodiversity hotspots where trade of wildlife species is most established, South China is the only region located in the northern hemisphere (Fig. 1d) and therefore is subject to the strong 'seesaw' effects of climate seasonality. Notably, SARS and also several outbreaks of imported MERS (Middle East respiratory syndrome), Asian flu in 1957 (H2N2), Hong Kong flu in 1968 (H3N2) and influenza A flu in 2009 (H1N1) were all linked to cold seasons and all also seeded in the northern hemisphere, not in tropical countries $^{17}$. The relevance of 'cold dry' weather for the progression of respiratory infections therefore seems crucial and independent of the geographical source of the spillover, an aspect that is often overlooked. For example, influenza epidemics tend to start in 'cold dry' conditions and 'humid rainy' conditions in temperate regions and tropical regions, respectively ${ }^{18}$. Still, the scientific literature on the subject has not fully established which are the relevant predictors of influenza seasonality, or why influenza epidemics peak differently in different regions (e.g., with unimodal, bimodal and year-round transmission ${ }^{19}$ ).

\section{Factors that affect respiratory infec- tions: lessons from COVID-19}

$\mathrm{AH}$ and/or temperature have been related to both outbreaks and the facilitated epidemic progression of influenza ${ }^{19}$. For temperature variations at the high end $\left(>30^{\circ} \mathrm{C}\right)$, a blockade of aerosol transmission in the case of influenza virus has been described, but controversy remains, because of the strong collinearity with humidity and unclear mechanistic associations ${ }^{20,21}$. Indeed, the 
drying time of a droplet and the growth rate of the spread of SARS-CoV-2 have also been found to be weakly related, at most. Apparently, SARS-CoV-2 has greater airborne survival and transmission than that of influenza virus in temperate regions. Evidence also suggests that SARS-CoV-2, as well as other coronaviruses, can be dispersed and potentially transmitted by aerosols.

However, the limited spread of SARS-CoV-2 in tropical countries might alternatively indicate that the high tropical $\mathrm{AH}$ values render the aerosolized route poorly effective. Fomite transmission can be a crucial pathway to consider instead in these settings, as enhanced $\mathrm{AH}$ and temperature render this pathway more effective. Therefore, in the tropics, viral viability and persistence in fomites on surfaces may be favored, which would enhance direct-contact and oral transmission if there is limited access to water and there are poor sanitation systems. Additionally, the large increases in $\mathrm{AH}\left(>3 \mathrm{~g} / \mathrm{m}^{3}\right)$ shown here for India and Africa (Fig. 1) may have an additional effect by decreasing the temporal stability of aerosols and therefore also restricting the window of time that virus-laden particles can persist suspended in air.

A fundamental role for climate in modulating the various COVID-19 pandemic waves that have occurred so far has been largely disputed ${ }^{22}$ and remains a challenging open question at this time. However, interestingly, a substantial difference in terms of epidemic progression surprisingly shows up if climate is added mechanistically in a process-based mathematical model for COVID-19. In the case of Japan, for example, explicit inclusion of temperature through the model's transmission term ( $\beta$ ) yields, as a fundamental difference, the current peak in winter 2020-2021 (Fig. 2c, changes in the active, recovered and death compartments). In the past, this model proved successful in simulating and forecasting the evolution of COVID-19 in different countries while accounting for a variety of public-health interventions ${ }^{23}$.

We present here results of the model fitted, for comparison, with and without climate (Fig. 2b,c). In one simulation, this $\beta$-parameter is a constant with no influence of external factors, while in the other, a seasonal temperature-dependent component $\beta(\mathrm{t})$ is added (Fig. $2 \mathrm{~b}, \mathrm{c}$ ). This term is inversely related to temperature and is normalized with the historical mean daily temperature from 1970 . In the sensitivity analysis here (Fig. 2b), most of the parameters in the model with seasonality are below a critical value, except for the temperature-dependent infection rate ' $\beta$ '. This rate achieves a much higher value (Fig. $2 \mathrm{~b}, \beta$ versus Temp) and thus denotes the high relevance of temperature forcing.

To sum up, without the effect of colder temperatures, but with the ongoing intervention measures and degree of lockdown maintained, Japan would probably not have experienced a third wave (Fig. 2c). Of course, uncertainties are large and are derived mostly from the limited data quality, but trends in the patterns are undeniably clear. Such a fundamental qualitative difference underscores the role of climate-and even more so, given its large public-health implications.

If, as for other $\beta$-coronaviruses (those that cause SARS and MERS) and wintertime respiratory viruses (e.g., rhinoviruses), a facilitating role is demonstrate for climate in the transmission of SARS-CoV-2, tailored environmental surveillance and a large-scale transdisciplinary modeling initiative would be justified.

Taking into account the role of human behavior in response to epidemics also adds an important and complex domain to be integrated into explicit modeling of climate. Specifically, during the 2003 SARS outbreak $^{24}$ and the 2009 A/H1N1 influenza pandemic ${ }^{25}$, people's awareness in Hong Kong and Beijing seemed to have effectively contributed to limiting the outbreak (e.g., compliance with precautionary actions such as the use of face masks, hand-washing, and social distancing in public transportation, as well as avoiding mass gatherings). At a time when the world is at stake with this global challenge, there is a critical need and, perhaps, a unique opportunity to assess the understanding of the environmental drivers of SARS-CoV-2 (and similar viruses) and their potential value in prediction. The traditional disciplinary divide between epidemiologists and climatologists needs to be effectively bridged during these hectic times.

\section{Xavier Rodó (D) 1,2凶, Adrià San-José2, Karin Kirchgatter ${ }^{\text {ID }}{ }^{3}$ and}

Leonardo López (ID) 2,4

${ }^{1}$ ICREA (Catalan Institute for Research and Advanced Studies), Barcelona, Spain. ${ }^{2}$ Climate and Health Program, ISGlobal, Barcelona, Spain. ${ }^{3}$ Departamento de Laboratórios EspecializadosSuperintendência de Controle de Endemias, Instituto de Medicina Tropical, Universidade de São Paulo, São Paulo, Brazil. ${ }^{4}$ Research Institute for Signals, Systems and Computational Intelligence, UNL-CONICET, Santa Fe, Argentina.

$\bigotimes_{e \text {-mail:xavier.rodo@isglobal.org }}$

Published online: 5 April 2021

https://doi.org/10.1038/s41591-021-01303-y

References

1. Njenga, M. K. et al. Am. J. Trop. Med. Hyg. 103 564-569 (2020).

2. Lone, S. A. \& Ahmad, A. Emerg. Microbes Infect. 9 , 1300-1308 (2020).

3. Zaitchik, B. F. et al. Nat. Commun. 11, 5730 (2020).

4. Plowright, R. K. et al. Nat. Rev. Microbiol. 15, 502-510 (2017)

5. Gortazar, C. et al. PLoS Pathog. 10, el004129 (2014).

6. Nunez, S., Arets, E., Alkemade, R., Verwer, C. \& Leemans, R. Clim. Change 154, 351-365 (2019).

7. Bartlow, A. W. et al. Vet. Sci. 6, 40 (2019).

8. dos Cunha, M. P. et al. Sci. Rep. 9, 20418 (2019).

9. Giovanetti, M. et al. J. Virol. 94, e01623-19 (2019).

10. Krishnamurthy, V. \& Goswami, B. N. J. Clim. 13, 579-595 (2000).

11. Hu, D. et al. Proc. Natl Acad. Sci. USA 113, E7730-E7739 (2016).

12. Mutreja, A. et al. Nature 477, 462-465 (2011).

13. Jenkins, C. N., Pimm, S. L. \& Joppa, L. N. Proc. Natl Acad. Sci. USA 110, E2602-E2610 (2013).

14. Johnson, C. K. et al. Proc. R. Soc. Lond. B 287, 20192736 (2020).

15. Rodó, X., Martinez, P., Siraj, A. \& Pascual, M. Nat. Commun. 12, 1555 (2021).

16. Wen, F., Bedford, T. \& Cobey, S. Proc. R. Soc. Lond. B 283, 20161312 (2016).

17. Noor, R. \& Maniha, S. M. Virusdisease 31, 441-449 (2020).

18. Shaman, J. \& Kohn, M. Proc. Natl Acad. Sci. USA 106, 3243-3248 (2009).

19. Lipsitch, M. \& Viboud, C. Proc. Natl Acad. Sci. USA 106, 3645-3646 (2009)

20. Lowen, A. C., Steel, J., Mubareka, S. \& Palese, P. J. Virol. 82, 5650-5652 (2008)

21. Tellier, R. Emerg. Infect. Dis. 12, 1657-1662 (2006).

22. Liu, X. et al. Environ. Res. 195, 110874 (2021).

23. López, L. \& Rodó, X. Nat. Hum. Behav. 4, 746-755 (2020).

24. Durham, D. P. \& Casman, E. A. J. R. Soc. Interface 9 , 562-570 (2012).

25. López, L., Fernández, M. \& Giovanini, L. MethodsX 7, 101030 (2020).

\section{Acknowledgements}

X.R. acknowledges support from the Spanish Ministry of Science and Innovation through the 'Centro de Excelencia Severo Ochoa 2019 2023' Program (CEX2018 000806S), and support from the Generalitat de Catalunya through the CERCA Program. A.S.J. was supported by a fellowship from 'la Caixa' Foundation, Spain (ID 100010434; fellowship code LCF/BQ/DR19/11740017).

Competing interests

The authors declare no competing interests. 